\title{
Impact of Inflammation-Metaplasia- Adenocarcinoma Sequence and Prevention in Surgical Rat Models
}

\author{
Tomoharu Miyashita $^{a}$ Furhawn A. Shah $^{d}$ Koichi Miwa $^{\mathrm{b}}$ Shozo Sasaki ${ }^{a}$ \\ Koji Nishijima $^{a}$ Katsunobu Oyama ${ }^{a}$ Itasu Ninomiya ${ }^{a}$ Sachio Fushida ${ }^{a}$ \\ Takashi Fujimura $^{\text {a }}$ Takanori Hattori $^{c}$ John W. Harmon ${ }^{d}$ Tetsuo Ohta ${ }^{a}$ \\ a Department of Gastroenterologic Surgery, Kanazawa University Hospital, Kanazawa, b Houju Memorial Hospital, \\ Nomi, and ' Department of Pathology, Shiga University of Medical Science, Otsu, Japan; ${ }^{\mathrm{d}}$ Department of Surgery, \\ Johns Hopkins Bayview Medical Center, Johns Hopkins University School of Medicine, Baltimore, Md., USA
}

\author{
Key Words \\ Histogenesis • Carcinogenesis • Barrett's esophagus • \\ Esophageal cancer $\cdot$ Esophageal adenocarcinoma . \\ Chemoprevention
}

\begin{abstract}
The incidence of esophageal cancer continues to rise in the Western world. Prior studies have suggested that gastroduodenal content reflux from gastroesophageal reflux disease induces the inflammation-mediated progression from hyperplasia to metaplasia, and to adenocarcinoma. We further investigated the sequential development of esophageal adenocarcinoma (EADC) with the use of an established surgical rat model. The present paper will describe the impact of the inflammation-metaplasia-adenocarcinoma sequence and chemoprevention in surgical rat models. A clinically relevant rat reflux model was used to investigate the cause of carcinogenesis, the sequential development of adenocarcinoma and chemoprevention with the use of a proton pump inhibitor. We found that duodenal reflux plays an important role in the inflammation-induced transformation of esophageal mucosa to adenocarcinoma. We were able to inhibit this transformation with rabeprazole, a proton pump inhibitor. Duodenal reflux promotes inflammation in the esophagus.
\end{abstract}

The inflammation-metaplasia-adenocarcinoma sequence is important in the progression and development of EADC. Carcinogenesis can be prevented with chemoprevention agents such as rabeprazole. These results will need to be validated in clinical trials.

Copyright $\odot 2013$ S. Karger AG, Basel

\section{Introduction}

The incidence of esophageal cancer continues to rise in the Western world $[1,2]$. The cause of the increase in incidence remains obscure and has prompted further investigation into this clinical problem. Prior studies have proposed that esophageal adenocarcinoma (EADC) develops through a sequence of events. Specifically, gastroduodenal content reflux from gastroesophageal reflux disease (GERD) induces the inflammation-mediated hyperplasia and metaplasia. This results in dysplasia and EADC [3]. The change in the diet and lifestyles in the Western world has contributed to an increase in the prevalence of obesity and hiatal hernias, and an increase in GERD. These lifestyle changes are thought to be responsible for the rise in the incidence of esophageal cancer [4].

\section{KARGER}

Fax +41613061234

E-Mail karger@karger.ch

www.karger.com
(C) 2013 S. Karger AG, Basel

0012-2823/13/0871-0006\$38.00/0

Accessible online at:

www.karger.com/dig
Tomoharu Miyashita

Department of Gastroenterologic Surgery

Kanazawa University Hospital, 13-1 Takaramachi

Kanazawa, Ishikawa 920-8641 (Japan)

E-Mail tomoharumiya@gmail.com 
Fig. 1. Diagram of the surgical procedure. The DGER model promotes regurgitation of both duodenal and gastric juice. The DER model produces regurgitation of duodenal juice alone. The GER model promotes regurgitation of gastric juice alone. The RY procedure does not result in duodenal or gastric regurgitation. The incidence of esophageal carcinoma (Ca.) in the DGER or the DER was significantly higher than in the GER or the RY ( $p<0.001)$. Esophageal carcinoma developed only in models with duodenal refluxate. No exogenous carcinogen was administered in this experiment.

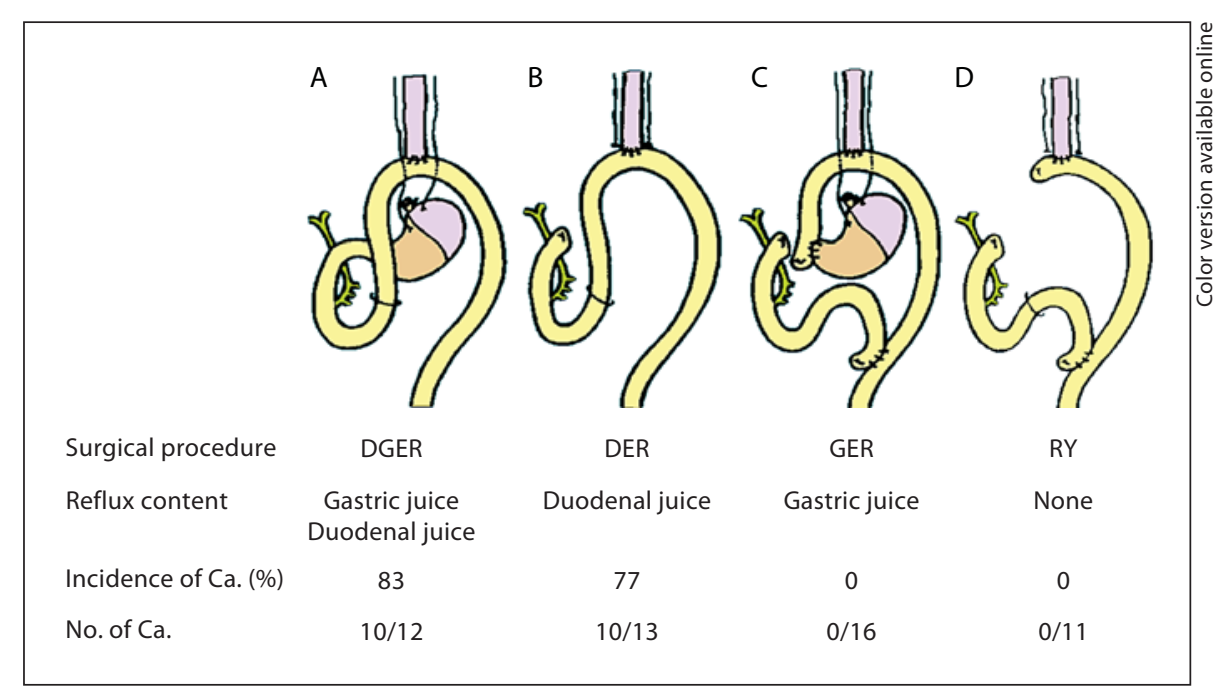

We investigated the sequential development of EADC using a surgical rat model. Prior studies have determined that duodeno-esophageal (DER) or duodeno-gastroesophageal reflux (DGER) in a rat model induced EADC $[5,6]$. These cancerous changes occurred without the use of any exogenous carcinogens. The rat model confirmed the histopathological sequence of events from GERD to EADC. Furthermore, a recent study established a relationship between the amount of reflux and the likelihood of developing EADC. The present paper will expand on prior reviews [7]. The goal is to describe the impact of the inflammation-metaplasia-adenocarcinoma sequence and chemoprevention in clinically relevant surgical rat models.

\section{Duodenal Content Reflux Induces Esophageal Carcinogenesis}

We established a surgical rodent model in which the forestomach was anastomosed with jejunum [5]. This duodeno-forestomach or duodeno-glandular-forestomach reflux model promoted gastro-duodenal reflux. The rodents subjected to this intervention developed squamous cell carcinoma, columnar epithelial metaplasia and mucinous adenocarcinoma. This was achieved without the use of carcinogens. Subsequent studies have confirmed these findings with alternate rodent models $[3,8]$.

The initial study suggested that duodenal reflux alone was sufficient to induce esophageal carcinogenesis $[6,8$, 9]. Other studies reported that a combination of gastric and duodenal contents was required to produce carcino- genesis. The importance of gastric reflux alone remained unclear.

This was further investigated with the development and use of four distinct rodent surgical models to determine the component responsible for carcinogenesis [6]. The DGER model promotes the reflux of both gastric and duodenal contents. The DER model consists of a total gastrectomy and anastomosis of the esophagus to the jejunum, thus producing reflux of duodenal contents alone. The gastro-esophageal reflux (GER) model includes the transposition of the duodenum distally. The model promotes gastric reflux alone. A Roux-en-Y (RY) procedure was developed to be used as a control with no reflux induction. Rodents in the duodenal reflux groups, DGER and DER, were the only rodents to develop esophageal carcinoma. The rodents not subjected to reflux or gastric reflux alone did not develop esophageal carcinoma (fig. 1). These findings have been confirmed by other subsequent studies. Furthermore, the study suggested that gastric juice may even be protective, since fewer rodents developed EADC in the DGER group than the DER group [10]. This may be secondary to alteration of the carcinogenic effect of bile compounds with exposure to an acid milieu.

We have established that duodenal reflux is important in the development of EADC. More recently, we concluded that there is an association between the severity of DER and the histological type of esophageal cancer. We found that increased duodenal content reflux promoted the development of EADC, while rodents subjected to mild duodenal content reflux were more likely to develop esophageal squamous cell carcinoma [11]. 
Fig. 2. Microscopic findings of DER in rat. a Metaplasia of the gastric foveolar epithelium was observed in the basal layer of the squamous epithelium, indicated by arrows. b Pyloric gland metaplasia with abundant mucus extending from the gastric foveolar epithelial metaplasia to the basal layer, indicated by arrows. c Completed columnar-lined metaplasia covered the entire mucosal layer, indicated by arrows, which is called Barrett's esophagus. Serial sections stained with GOS (d), Con A (e) or HID-AB (f). In addition to gastric foveolar epithelial and pyloric gland metaplasia, intestinal epithelial metaplasia was observed. g, h Representative photomicrographs of esophageal carcinoma that resulted from surgically induced gastroduodenal reflux: adenocarcinoma (g), squamous cell carcinoma with keratin pearls (h).
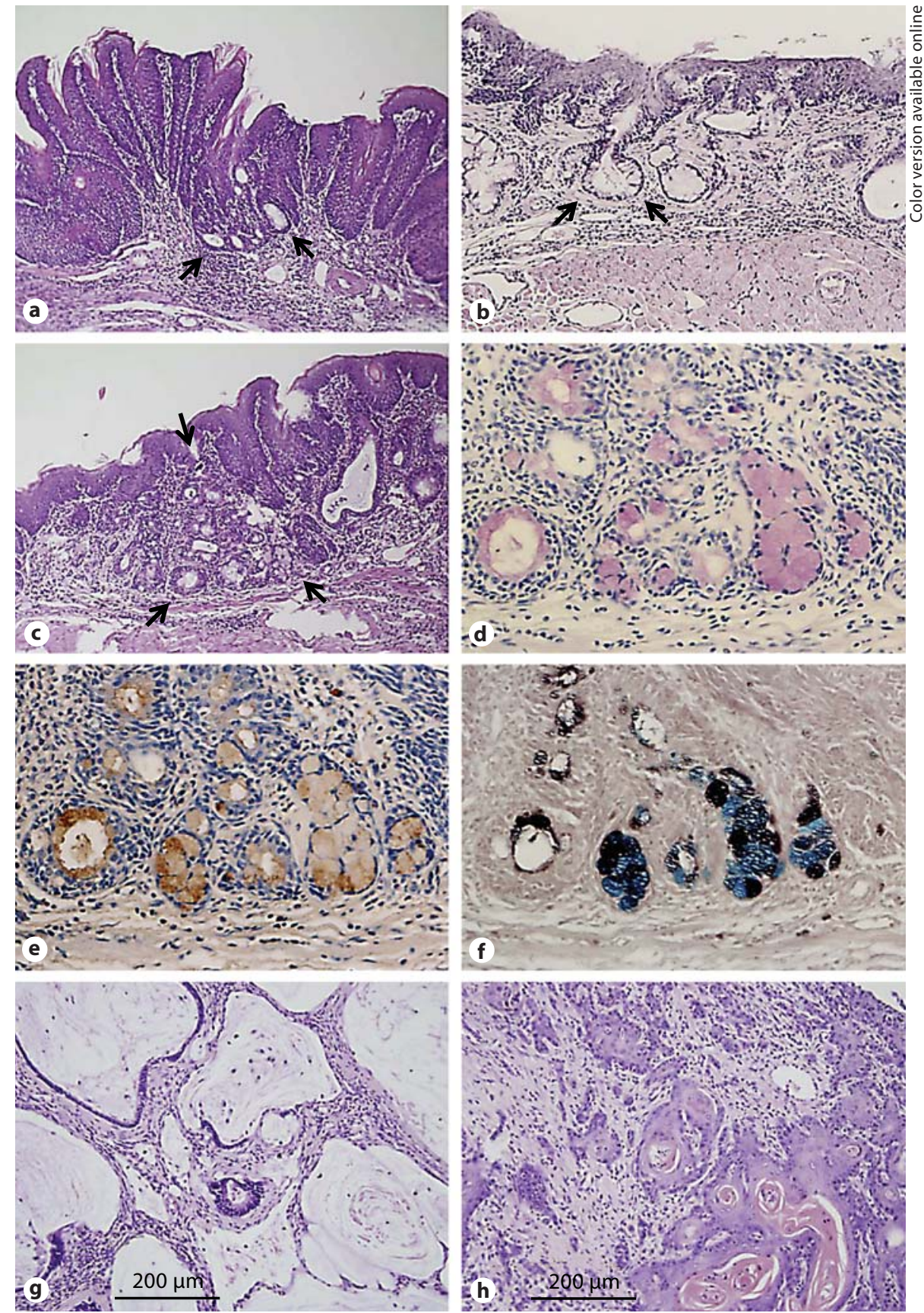

\section{Inflammation-Metaplasia-Adenocarcinoma Sequence in a Rat Model}

A rodent surgical reflux model was developed to further investigate the pathogenesis of esophageal cancer [8]. The DER model led to the exposure of the esophageal mucosa to duodenal contents. This exposure resulted in an inflammatory response. The animals were sacrificed post-surgically at 10 -week intervals for a total of 50 weeks. Within 10 weeks of surgery, the squamous epithelium was infiltrated with lymphocytes. There were signs of proliferative squamous hyperplasia, regenerative thickening and basal cell hyperplasia. In 20 weeks, glandular structures were seen throughout the basal layer of the esophageal epithelium. This was followed with proliferative squamous hyperplasia and erosions near the anasto- 
Fig. 3. Inflammation-metaplasia-dysplasia-adenocarcinoma sequence in a surgical reflux model. DER or DGER induces severe inflammation. Esophageal squamous epithelium regenerated the proliferative hyperplasia. Proliferative hyperplasia is replaced with metaplasia, such as gastric foveolar, gastric pyloric and intestinal metaplasia. These metaplasias, including Barrett's metaplasia, progress to dysplasia and adenocarcinoma. Rabeprazole impedes the development of Barrett's metaplasia and esophageal cancer.

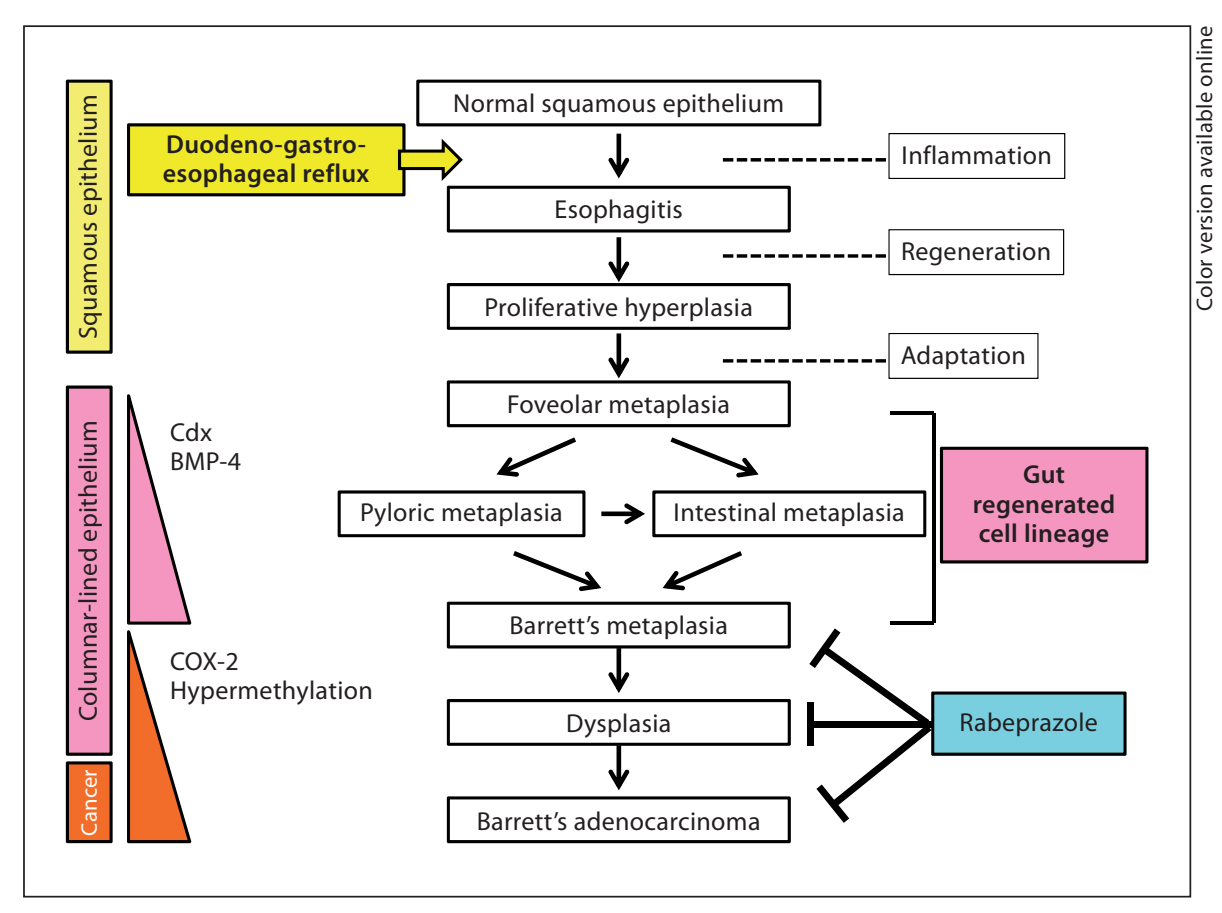

mosis site. The basal layer of esophageal squamous epithelium developed galactose oxidase-Schiff (GOS)-positive glandular structures and gastric foveolar metaplasia (fig. 2a). These histological changes set the stage for the development of esophageal carcinoma.

The rat model displayed sequential changes in the esophageal epithelium surrounding the anastomosis. There were significant metaplastic changes to the gastric glands in rodents sacrificed at 30 weeks. The glands developed deep within the mucosa and formed cysts with gastric pyloric glandular metaplasia (fig. 2b). Glandular tissue was present throughout the entire esophageal mucosa and advanced to the luminal side of the digestive tract. The squamous epithelium was completely replaced with dysplastic epithelium (fig. 2c). These glands stained for GOS (fig. 2d), concanavalin A (Con A; fig. 2e) and high iron diamine and alcian blue (HID-AB; fig. 2f), which are known markers for Barrett's esophagus in humans. Thus, the sequence of events in the rat model correlates with the human condition. Over 40 weeks, at the time of sacrifice and study conclusion, EADC (fig. 2g) was seen near the esophago-jejunal junction at the site of anastomosis. The regions of EADC are surrounded with areas of columnar-lined epithelium. The proximal esophagus displayed esophageal squamous cell carcinoma (fig. 2h) and adenosquamous carcinoma.

Inflammation-Metaplasia-

Adenocarcinoma Sequence in Rat Models
Bilious substances are toxic to the epithelium. The natural response of esophageal tissue to toxic substances such as bilious reflux is inflammatory metaplasia. This is an adaptive response to protect the epithelium, since metaplastic tissue is more resistant to change than natural tissue. Recent studies determined that the metaplastic changes are driven by increased expression of the homeobox genes related with intestinal development (Cdx2) [12]. These genes are activated by bile acids such as deoxycholic acid and cholic acids $[13,14]$. Souza et al. [15] summarized the pathogenesis of inflammation-induced metaplasia. The study hypothesized that acid and gastric juices breakdown the tight junctions between esophageal squamous epithelial cells and result in increased epithelial permeability. These structural changes allow acid, bile salts and other inflammatory mediators to penetrate into the deep layer of esophageal mucosa. The interaction of these compounds with the esophageal mucosa is thought to trigger the expression of $\mathrm{Cdx} 1, \mathrm{Cdx} 2$ and other genes that induce the intestinalization of esophageal tissue. The expression of BMP4 and other morphogenic factors increase in stroma cells [16]. The authors suggest that the interaction between Cdx genes and BMP- 4 mediates the development of Barrett's metaplasia. A different set of genes are responsible for CpG island hypermethylations and the development of Barrett's esophagus into EADC [17]. Thus, the se- 
Table 1. Histopathological findings

\begin{tabular}{lcc}
\hline & $\begin{array}{l}\text { Placebo } \\
\text { group }\end{array}$ & $\begin{array}{l}\text { Rabeprazole } \\
\text { group }\end{array}$ \\
\hline Total surgeries & 38 & 38 \\
Survivors & 23 & 17 \\
Average weight \pm SE, g & $292 \pm 58$ & $265 \pm 43$ \\
Cancer & $17(74 \%)$ & $5(29 \%)$ \\
Adenocarcinoma & 14 & 5 \\
Squamous cell carcinoma & 3 & 2 \\
Barrett's metaplasia & $23(100 \%)$ & $11(65 \%)$ \\
Proliferative hyperplasia & $23(100 \%)$ & $17(100 \%)$ \\
\hline
\end{tabular}

The incidence of cancer ( $p<0.05, \chi^{2}$ test) and Barrett's metaplasia ( $p<0.05$, Fisher's exact test) was lower in the rabeprazole group as compared to the placebo group. Two animals developed both squamous cell carcinoma and adenocarcinoma.

quence of several events progressing from DEGR to EADC is thought to involve the development of inflammation-stimulated proliferative squamous hyperplasia and metaplasia such as in Barrett's esophagus, followed by multifocal and EADC (fig. 3).

\section{Chemoprevention for Barrett's Metaplasia and Adenocarcinoma}

We have established that significant bilious reflux increases the likelihood of EADC in a rodent reflux model. This model allowed us to investigate the response to chemopreventive agents.

We evaluated the effectiveness of the proton pump inhibitor rabeprazole as a chemoprevention agent in a surgical rat reflux model of esophageal cancer [18]. We found that rabeprazole significantly reduced the incidence of Barrett's metaplasia and esophageal cancer (table 1). We hypothesized that the proton pump inhibitor raised $\mathrm{pH}$ and altered the interactions between duodenal and gastric milieu. This led to the production of substances that promoted inflammatory changes. These substances may be bile acids altered by the milieu or even pancreatic enzymes. Alternatively, rabeprazole may possess anti-inflammatory properties that protect the esophageal mucosa.

Prior epidemiological studies revealed long-term use of proton pump inhibitors decreased the incidence of dysplasia and adenocarcinoma in patients with an established diagnosis of Barrett's esophagus [19, 20]. However, the data remains mixed. Another large review compared the efficacy of anti-reflux surgery with chemotherapy in the prevention of progression of Barrett's esophagus to EADC. The study found that surgery decreased the likelihood of progression of Barrett's esophagus to EADC and increased the likelihood of regression of Barrett's esophagus [21]. A more recent trial to evaluate the effects of omeprazole in combination with aspirin on the rate of progression to high-grade dysplasia or EADC in patients with Barrett's esophagus is ongoing. Preliminary results suggest a synergistic anti-inflammatory effect [22].

\section{Conclusions}

The use of a clinically relevant surgical rodent reflux model allowed us to better understand the pathogenesis of esophageal adenocarcinoma. The model was used to verify the importance of duodenal contents reflux in the promotion of esophageal cancer. Inflammation was found to be the key component of the transformation of the esophageal mucosa to adenocarcinoma. Chemoprevention with rabeprazole was suggested to provide an anti-inflammatory and protective effect. While the results obtained from our animal model are impressive, they require further investigation and verification with human clinical trials.

\section{Disclosure Statement}

The authors declare that no financial or other conflicts of interest exist in relation to the content of this article.

References

1 Landis SH, Murray T, Bolden S, Wingo PA: Cancer statistics, 1998. CA Cancer J Clin 1998;48:6-29.

-2 Landis SH, Murray T, Bolden S, Wingo PA: Cancer statistics, 1999. CA Cancer J Clin 1999;49:8-31.

3 Fein M, Peters JH, Chandrasoma P, Ireland AP, Oberg S, Ritter MP, Bremner CG, Hagen JA, DeMeester TR: Duodenoesophageal reflux induces esophageal adenocarcinoma without exogenous carcinogen. J Gastrointest Surg 1998;2:260-268.

-4 Lagergren J, Bergström R, Lindgren A, Nyrén O: Symptomatic gastroesophageal reflux as a risk factor for esophageal adenocarcinoma. N Engl J Med 1999;340:825-831.

$\checkmark 5$ Miwa K, Segawa M, Takano Y, Matsumoto H, Sahara H, Yagi M, Miyazaki I, Hattori T: Induction of oesophageal and forestomach carcinomas in rats by reflux of duodenal contents. Br J Cancer 1994;70:185-189.
10

Digestion 2013;87:6-11
Miyashita et al. 
6 Miwa K, Sahara H, Segawa M, Kinami S, Sato T, Miyazaki I, Hattori T: Reflux of duodenal or gastro-duodenal contents induces esophageal carcinoma in rats. Int J Cancer 1996;67: 269-274.

$>7$ Fujimura T, Oyama K, Sasaki S, Nishijima K, Miyashita T, Ohta T, Miwa K, Hattori T: Inflammation-related carcinogenesis and prevention in esophageal adenocarcinoma using rat duodenoesophageal reflux models. Cancers 2011;3:3206-3224.

-8 Miyashita T, Ohta T, Fujimura T, Ninomiya I, Fushida S, Hattori T, Miwa K: Duodenal juice stimulates oesophageal stem cells to induce Barrett's oesophagus and oesophageal adenocarcinoma in rats. Oncol Rep 2006;15: 1469-1475.

$\checkmark 9$ Pera M, Brito MJ, Poulsom R, Riera E, Grande L, Hanby A, Wright NA: Duodenalcontent reflux esophagitis induces the development of glandular metaplasia and adenosquamous carcinoma in rats. Carcinogenesis 2000;21:1587-1591.

-10 Ireland AP, Peters JH, Smyrk TC, DeMeester TR, Clark GW, Mirvish SS, Adrian TE: Gastric juice protects against the development of esophageal adenocarcinoma in the rat. Ann Surg 1996;224:358-370.
11 Miyashita T, Miwa K, Fujimura T, Ninomiya I, Fushida S, Shah FA, Harmon JW, Hattori T, Ohta T: The severity of duodeno-esophageal reflux influences the development of different histological types of esophageal cancer in a rat model. Int J Cancer 2012, Epub ahead of print

12 Tatsuta T, Mukaisho K, Sugihara H, Miwa K, Tani T, Hattori T: Expression of Cdx2 in early GRCL of Barrett's esophagus induced in rats by duodenal reflux. Dig Dis Sci 2005;50: 425-431.

13 Marchetti M, Caliot E, Pringault E: Chronic acid exposure leads to activation of the $\mathrm{cdx} 2$ intestinal homeobox gene in a long-term culture of mouse esophageal keratinocytes. J Cell Sci 2003;116:1429-1436.

Kazumori H, Ishihara S, Rumi MA, Kadowaki Y, Kinoshita Y: Bile acids directly augment caudal related homeobox gene $\mathrm{Cdx} 2$ expression in oesophageal keratinocytes in Barrett's epithelium. Gut 2006;55:16-25.

15 Souza RF, Krishnan K, Spechler SJ: Acid, bile, and CDX: the ABCs of making Barrett's metaplasia. Am J Physiol Gastrointest Liver Physiol 2008;295:211-218.

16 Wang DH, Clemons NJ, Miyashita T, Dupuy AJ, Zhang W, Szczepny A, CorcoranSchwartz IM, Wilburn DL, Montgomery EA, Wang JS, Jenkins NA, Copeland NA, Harmon JW, Phillips WA, Watkins DN: Aberrant epithelial-mesenchymal Hedgehog signaling characterizes Barrett's metaplasia. Gastroenterology 2010;138:1810-1822.
7 Sato F, Meltzer SJ: CpG island hypermethylation in progression of esophageal and gastric cancer. Cancer 2006;106:483-493.

18 Miyashita T, Shah FA, Marti GP, Wang J, Bonde P, Gibson MK, Ohta T, Montgomery EA, Duncan M, Harmon JW: Rabeprazole impedes the development of reflux-induced esophageal cancer in a surgical rat model. Dig Dis Sci 2011;56:1309-1314.

19 El-Serag HB: The epidemic of esophageal adenocarcinoma. Gastroenterol Clin North Am 2002;31:421-440.

20 Hillman LC, Chiragakis L, Shadbolt B, Kaye GL, Clarke AC: Proton-pump inhibitor therapy and the development of dysplasia in patients with Barrett's oesophagus. Med J Aust 2004; 180:387-391.

21 Csendes A, Bragheto I, Burdiles P, Smok G, Henriquez A, Parada F: Regression of intestinal metaplasia to cardiac or fundic mucosa in patients with Barrett's esophagus submitted to vagotomy, partial gastrectomy and duodenal diversion: a prospective study of 78 patients with more than 5 years of follow up. Surgery 2006;139:46-53.

$\checkmark 22$ Das D, Chilton AP, Jankowski JA: Chemoprevention of oesophageal cancer and the AspECT trial. Recent Results Cancer Res 2009;181:161-169. 